\begin{tabular}{lllllllllllllllllllllllllllllllll}
\hline$R$ & $E$ & $V$ & I & S & T & A & D & E & E & S & T & U & D & I & O & S & I & N & T & E & R & N & A & C & I & O & N & A & L & E & S
\end{tabular}

\title{
APEC 2004: "viejas" y nuevas dinámicas de apertura económica transpacífica
}

\section{Hernán Gutiérrez B.}

En 2004, el foro de Cooperación Económica de Asia-Pacífico o APEC, según su sigla en inglés, cumplirá 15 años desde que fue creado en Canberra, como resultado de los esfuerzos de australianos y japoneses por impedir el quiebre de la región de Asia-Pacífico en dos grupos o bloques económicos: Norteamérica, liderado por los Estados Unidos y Asia oriental, impulsado por Japón y las economías más avanzadas del noreste de Asia.

Los miembros del foro, inicialmente formado por 12 paises ${ }^{1}$, han aumentado en número con las llamadas economías que lo integran, así como en el nivel de influencia política, la profundidad del programa económico y lo ambicioso de sus metas. El propósito de este artículo es describir y evaluar el proceso del APEC en uno de sus dos pilares tradicionales, la liberalización y facilitación del comercio y de las inversiones, y en especial los temas de comercio internacional. En particular, nos interesa analizar su aproximación a los Tratados de Libre Comercio (TLC), por cuanto estos fueron, justamente, uno de los factores que gatillaron la creación del APEC, por una parte, y porque la cara más visible de la apertura transpacífica ha sido, además de la apertura unilateral, la liberalización y facilitación del comercio a través de los TLC. Además de los hitos del APEC en política comercial y su relación con el proceso de apertura económica multilateral, analizaremos el tema de los TLC desde la perspectiva de las estrategias comerciales como pieza central de las políticas económicas en Asia nororiental, y la relación de los TLC con la economía política regional.

${ }^{1}$ Estados Unidos, Canadá, Japón, Corea del Sur, Tailandia, Malasia, Indonesia, Singapur, Brunei Darussalam, Filipinas, Australia, y Nueva Zelandia. Posteriormente, los miembros del APEC serían denominados economías, debido al ingreso simultáneo, en 1991, de China, China-Hong Kong y ChinaTaipei. Además de las "tres chinas", ingresarían Papua-Nueva Guinea y México, en 1992, Chile, en 1994, y la Federación de Rusia, Perú y Vietnam, en 1998. 


\section{El APEC y LA NUEVA GENERACIÓN DE} ORGANISMOS REGIONALES

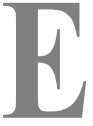

El APEC pertenece a una nueva generación de organismos regionales de integración económica, que nace y crece en un escenario mundial inédito. La densidad y calidad del intercambio comercial y financiero a lo ancho del globo terráqueo, productos de la economía global y de la información ${ }^{2}$, generan una interdependencia compleja entre naciones pertenecientes a civilizaciones muy diferentes, cualquiera sea su tamaño y grado de apertura económica, y cualquiera sea su grado de desarrollo económico, político y social. La densidad de la interfase económica internacional supera ampliamente el plano más simple del intercambio de insumos productivos y de bienes finales, en el terreno comercial, y de los flujos de inversión extranjera, sólo atraídos por el acceso a los mercados internos, en el plano de las inversiones productivas. El despliegue de una estrategia global de investigación, producción y distribución por parte de las empresas transnacionales ha fomentado el crecimiento exponencial del comercio intra- industrial y de los servicios. Las economías de industrialización reciente, tanto en Asia como en América Latina y Europa, alteraron la estratificación mundial y la distribución del poder económico ${ }^{3}$. Las relaciones entre los agentes del comercio $\mathrm{y}$ de las finanzas internacionales se han ampliado hacia las alianzas estratégicas y las fusiones, introduciendo una nueva dimensión a la tradicional relación entre compradores y vendedores, y a la adopción del sistema industrial fordista.

\section{Las economías de industrialización reciente alteraron la estratificación mundial y la distribución del poder económico.}

El APEC, más que cualquier otro organismo económico regional, es un espejo de esta nueva realidad ${ }^{4}$. Es antes que nada un foro de cooperación, apoyado por una burocracia reducida, que trabaja en el marco de una institucionalidad flexible y descentralizada. El APEC es un proceso que avanza sobre la base de las convergencias y los consensos que contribuyen a profundizar la liberalización y la

\footnotetext{
${ }^{2}$ Manuel Castells desarrolla un excelente y completo análisis de las particularidades de la integración en el este de Asia en el marco de la globalización, junto a los desafíos sistémicos que le planteó la crisis de 1997/1998. Ver La Era de la Información: Economía, Sociedad y Cultura, Madrid, Siglo XXI, 1999; Vol III, capítulo 4, pp. 235-342.

${ }^{3}$ James Rosenau realiza una acertada caracterización de las nuevas dinámicas de las relaciones internacionales, en el marco de la globalización, en Turbulence in World Politics, Princeton, Princeton University Press, 1991; especialmente el capítulo 1, pp. 3-20.

${ }^{4}$ Richard Feinberg hace un documentado análisis de la génesis, proceso institucional y contenido de la agenda del APEC, contrastándola con el Área de Libre Comercio de las Américas (ALCA), desde la perspectiva de los nuevos regionalismos. Ver "Análisis Comparativo de Integración Regional en un Caso de Mellizos No Idénticos: APEC y el ALCA”, Integración y Comercio, № 10, enero-abril 2000, pp. 3-34.
} 
facilitación del comercio y de las inversiones a nivel de la economía regional y también mundial. Su objetivo, conocido como las Metas de Bogor, es constituir en Asia-Pacífico un espacio económico abierto, enmarcado en los principios de la Organización Mundial de Comercio (OMC), que se extienda a economías no pertenecientes al APEC. A lo largo de su vida, el foro ha cristalizado este proceso en el concepto de regionalismo abierto y en 1994, en Bogor (Indonesia), se propuso alcanzar la meta del libre comercio regional en 2010, en el caso de las economías desarrolladas y en 2020 en el de las economías en desarrollo.

\section{El APEC avanza a partir de convergencias y consensos.}

El concepto de regionalismo abierto, fue acuñado en $1992^{6}$ en la "Declaración de San Francisco" aprobada por el Consejo de Cooperación Económica del Pacífico (PECC), que reconoce que el dinamismo económico de la región se debe principalmente a su creciente apertura económica y al respaldo a la liberalización multilateral ${ }^{7}$. En el APEC el concep- to adquirió fuerza y se tornó polémico con el segundo informe elaborado por un grupo especial de personalidades, el Grupo de Personas Eminentes del APEC, en 1994, que establece que el concepto debe ser puesto en práctica mediante una fórmula compuesta de cuatro elementos: 1 . la máxima extensión y profundidad posibles de la apertura unilateral; 2. el compromiso de seguir abriendo la economía a los países no miembros; 3 . en voluntad de extender la liberalización regional sobre una base de reciprocidad, mientras que liberaliza internamente a partir del principio de la nación más favorecida; y 4. el reconocimiento de que en el marco del APEC cualquiera de sus miembros puede ampliar unilateralmente su liberalización en forma condicional o incondicional $^{8}$. Se ha definido el regionalismo abierto del APEC como un regionalismo que pone énfasis simultáneamente en un enfoque regional sobre la liberalización y en el respeto por el principio de la nodiscriminación. Desde esta perspectiva, la cuestión clave del regionalismo abierto consiste en que el APEC no comenzaría definiéndose a sí mismo como un bloque comercial preferencial.

A lo largo de la última década y me-

${ }^{5}$ Robert Scollay, "Intersecciones complejas: Los lazos comerciales entre Asia del Este y América Latina en el marco de recientes desarrollos multilaterales, regionales y subregionales", Integración y Comercio, $\mathrm{N}^{\mathrm{o}} 12$, septiembre-diciembre 2000, pp. 25-47. Ver también, Mikio Kuwayama, Open Regionalism in Asia Pacific and Latin America: A Survey of the Literature, CEPAL, Serie Comercio Internacional, $\mathrm{N}^{\circ}$ 4, diciembre 1999.

${ }^{6}$ El concepto en sí no es nuevo. Fue ocasionalmente utilizado a fines de los años sesenta por un grupo de académicos japoneses, y formulado por primera vez en el ámbito político, a fines de los años setenta, por el Primer Ministro japonés, Masayoshi Ohira. Mario Artaza Rouxel, "Estructura institucional y normas de funcionamiento de APEC", DIRECON, 2003.

${ }^{7}$ Juan Salazar, Chile y la Comunidad del Pacifico, Santiago, Editorial Universitaria, 1999, Tercera Parte, "La Comunidad del Pacífico: La inserción de Chile en el proceso de cooperación regional”; p. 183.

${ }^{8}$ Kuwayama, op. cit., 1999; p. 12. 
dia, el APEC ha ideado mecanismos novedosos para poner en práctica su conceptualización del regionalismo abierto a través del unilateralismo concertado, el cual reconoce que la liberalización no discriminatoria voluntaria remite a la liberalización unilateral pero reconoce también que el apoyo mutuo, la creación de confianza y la presión de los pares brindada por la concertación regional pueden efectuar una contribución importante para sustentar el compromiso de liberalización a nivel de la economía mundial ${ }^{9}$. A diferencia de otros organismos que negocian acuerdos vinculantes, el APEC se basa en modalidades que promuevan la concertación voluntaria, traducida en acciones unilaterales, las que en el APEC se conocen como unilateralismo concertado. Los instrumentos para avanzar en esta dirección se definieron en 1995, un año después de que se fijaran las metas de Bogor en la Reunión Anual de los Líderes del APEC, en lo que se conoce como la Agenda de Acción de Osaka. Uno de sus pilares está constituido por los Planes de Acción Individuales (IAP, por su sigla en inglés), que son básicamente un ejercicio periódico de evaluación de los compromisos individua- les de liberalización y facilitación comercial y financiera ${ }^{10}$. En la práctica, estos son réplicas, adaptadas a la cultura transpacífica de las negociaciones comerciales, de los ejercicios de revisión de las políticas comerciales de la OMC. Por otra parte, los Planes de Acción Colectivos (CAP, según su sigla en inglés), en temas propios de la integración profunda de las economías como, por ejemplo, las políticas de competencia, la transparencia y las compras públicas, completan el segundo pilar de las actividades del foro en materia de comercio y finanzas internacionales. En su conjunto, ambos sostienen las actividades del APEC en su agenda de liberalización y facilitación comercial y financiera, o TILF, según su sigla en inglés.

\section{El regionalismo abierto del APEC pone énfasis en un enfoque regional de la liberalización.}

Pese a las críticas y a sus manifiestas deficiencias ${ }^{11}$, el balance del APEC mues-

${ }^{9}$ Scollay, op. cit., 2000; p. 28.

${ }^{10}$ Refiriéndose al APEC, Miles Khaler plantea la hipótesis del arco de información: las instituciones descentralizadas e informales pueden sugir en entornos en que la información es escasa y cara, como en las economías del este de Asia, que requiere de una compilación sustancial de información antes de embarcarse en diseños institucionales más complejos. Ver "El nuevo regionalismo y sus instituciones", en Roberto Bouzas (ed.), Regionalización e Integración Económica: Instituciones y Procesos Comparados, Buenos Aires, GEL, 1997; pp. 27-55. Darby Higgs también realiza un interesante análisis del APEC desde una perspectiva sociológica, como sistema, en "APEC as a complex adaptative system: Insights on the problem of multilateralism versus bilateralism from a new science", trabajo presentado en la conferencia anual del Consorcio Internacional de Centros de Estudios APEC, Tianjin, 2001.

${ }^{11}$ La red internacional de evaluadores de APIAN (sigla en inglés que corresponde a Red Internacional de Evaluación del APEC), ha producido una serie de incisivos estudios sobre la institucionalidad, agenda y proceso de APEC. A sus informes se puede acceder en: <www.apian.org>. 
tra que el foro ha superado las expectativas que se forjaron al momento de su creación, en 1989, en Canberra (Australia), como un espacio de diálogo continuo entre altos funcionarios y ministros sobre una agenda abierta de temas económicos. A lo largo de los años se ha fortalecido en diversos planos. Primero, mediante el incremento del número de economías miembros, convirtiéndose, de paso, en el primer organismo económico intergubernamental que incluye a las "tres Chinas". El APEC ha crecido hasta abarcar cerca de $60 \%$ del producto mundial y una cifra similar del comercio internacional, reuniendo polos gravitantes de innovación tecnológica y científica en América, Asia oriental y Oceanía. Su perfil político recibió en 1993 el impulso de las llamadas Reuniones Anuales de los Líderes del $\mathrm{APEC}^{12}$, a partir de la reunión realizada en Blake Island, Estados Unidos.

Uno de los fenómenos que definen la globalización es la emergencia de un mapa más complejo, compuesto de más estratos de naciones que superan la dicotomía norte-sur que imperó en la década de los años setenta y principios de los ochenta. El APEC es un foro sobre política comercial y un espacio para la cooperación técnica y económica, una de cuyas características más interesantes es, precisamente, la de ser antesala de las negociaciones multilaterales que reúnen a las principales naciones industrializadas -exceptuando las de Europa- con economías de industrialización reciente y economías en vías de desarrollo. Reúne a países con sólidas economías de mercado junto con naciones de economía mixta en plena y dificultosa transición. Al respecto, la membresía de China es paradigmática.

\section{El APEC es el primer organismo económico intergubernamental que incluye a las "tres Chinas".}

Una institucionalidad flexible, basada en una pequeña burocracia, en reuniones periódicas de redes de expertos, altos oficiales y ministros, coronadas por la Reunión de los Líderes del APEC, ha permitido que el foro construya importantes consensos regionales en favor de la reducción de las barreras arancelarias y de las medidas paraarancelarias. Al mismo tiempo, ha canalizado intercambios sobre una amplia agenda de temas y actividades que cubren todos los aspectos del intercambio económico. Los grupos de trabajo del APEC abarcan en toda su extensión y profundidad los temas propios de la integración profunda ${ }^{13}$ incluidos en los acuerdos comerciales más ambiciosos. Su ámbito de acción se extendió, en la última década, hacia la cooperación econó-

${ }^{12}$ Las llamadas Reuniones Informales de Líderes del APEC reúnen a los mandatarios de las economías del foro, y se llaman así para explicitar que no tienen carácter de oficiales, debido a la membresía de la República Popular China, de China-Taipei y de China-Hong Kong.

${ }^{13}$ Ésta incluye, además de los temas comerciales tradicionales, el comercio de servicios, movimiento de factores, armonización de regímenes regulatorios y, en definitiva, todas las políticas nacionales que se estima pueden afectar la competitividad internacional. Ver, Kuwayama, 1999, op. cit.; pp. 32-34. 
mica y técnica, o ECOTECH, según su sigla en inglés. El APEC ha sido calificado de "sistema evolutivo de diplomacia económica multilateral basado en los consensos entre los Estados (economías) participantes" $" 14$.

\section{Los grupos de trabajo del APEC abarcan los temas propios de la integración profunda.}

\section{Los primeros años: multilateralismo y regionalismo abierto}

El regionalismo del APEC, desde su concepción, se inscribe explícitamente en el marco del fortalecimiento del sistema multilateral de comercio, bajo la égida del GATT, primero, y de la OMC después. Ciertamente, el proceso APEC ha estado estrechamente relacionado con las negociaciones multilaterales de apertura comercial y financiera, si bien en el tiempo esta relación ha sido compleja y cambiante.
En una primera fase, que va desde la creación del foro y se extiende hasta la Reunión Informal de los Líderes realizada en Manila, Filipinas, en 1996, el gran mérito del APEC fue encauzar la interdependencia transpacífica y mantener espacios de diálogo y cooperación en un ambiente marcado en los primeros años por las dificultades que enfrentaba la Ronda Uruguay y por las incertidumbres que imperaban acerca del nuevo escenario económico mundial que surgiría de la posguerra fría ${ }^{15}$. En esta primera fase, el foro se nutrió de los temores de que surgieran bloques económicos debido al fortalecimiento y ampliación de la integración europea, junto con la integración comercial y productiva de Asia oriental y con el inédito regionalismo estadounidense expresado, primero, en la Iniciativa para las Américas, y luego en el Acuerdo de Libre Comercio de América del Norte (NAFTA). El APEC permitió neutralizar y desanimar las propuestas tendientes a formar un bloque de economías de Asia oriental, impulsado principalmente por Malasia entre las economías del sudeste asiático $^{16}$, diluyendo el espectro de fric-

${ }^{14}$ Manfred Wilhelmy y Rosa María Lazo desarrollan un completo y documentado análisis de los orígenes de APEC, desde la perspectiva del regionalismo en el Pacífico asiático, incluyendo la dinámica de otras entidades regionales como el Consejo de Cooperación Económica del Pacífico (PECC, por su sigla en inglés). Ver, "La Política Multilateral de Chile en Asia Pacífico", Estudios Internacionales, N ${ }^{\circ}$ 117, Año XXX, enero-marzo DE 1997; pp. 3-35.

${ }^{15}$ Hernán Gutiérrez B., “APEC: Regionalismo abIerto y globalización”, Diplomacia, septiembreoctubre DE 1997; pp. 18-23.

${ }^{16}$ Manfred Wilhelmy y Hernán Gutiérrez B., "Concepciones Latinoamericanas y Asiáticas sobre Cooperación Regional”, en Manfred Mols, Manfred Wilhelmy y Hernán Gutiérrez B., América Latina y el Sudeste Asiático. Perfiles de Cooperación Regional, Santiago, Colección Estudios Internacionales, Instituto de Estudios Internacionales, Universidad de Chile, 1995; pp. 229-270. Ver también, Juan José Ramírez Bonilla, "La región del Pacífico ante los efectos disruptores de los acuerdos de libre comercio", V Reunión de la Red de América Latina y el Caribe de Centros de Estudios sobre el Pacífico, Buenos Aires, 12 y 13 de diciembre de 2002; p. 3. 
ciones comerciales entre los grandes bloques en 1991, en la Reunión Ministerial de Seúl (en ese entonces máxima instancia del APEC). Facilitó, en segundo lugar, la discusión a nivel regional de la agenda multilateral, integrando a dos de las principales economías del mundo, los Estados Unidos y Japón, junto con las economías asiáticas de industrialización reciente, incluyendo a las "tres Chinas".

\section{El impacto de la crisis asiática en el} APEC

La crisis productiva y financiera de 1997-1998 se tradujo en la adopción de nuevos lineamientos estratégicos en las áreas más relevantes de las políticas económicas que se aplican en gran parte de las economías asiáticas, apartándolas de manera significativa de los postulados clásicos de las políticas industriales aplicadas en las tres últimas décadas. Las transformaciones estructurales han sido particularmente profundas en las economías del noreste de Asia.

En el plano internacional, la crisis económica que afectó al este de Asia alteró el escenario regional y mundial, y en definitiva contribuyó a aumentar la estatura de China, producto del liderazgo que ejerció al mantener la fortaleza su mone$\mathrm{da}$, el renmimbi. La mayor credibilidad de su economía en el concierto internacional le proporcionó una base más segu- ra para encarar las complejidades de la interdependencia, una realidad nueva para la economía china, que se caracterizó por su alto grado de autarquía, pero en la que ya a fines de la década de los años noventa el comercio exterior representaba cerca del $45 \%$ del PIB. Fue, además, un elemento político que amplió sus apoyos externos en las negociaciones para acceder a la OMC, estancadas desde que presentara su candidatura al GATT, en 1986.

\section{Se adoptaron lineamientos estratégicos distintos de los postulados clásicos de las políticas industriales de los últimos decenios.}

La crisis asiática repercutió en el APEC a través del estancamiento del proceso de apertura regional, hecho que se manifestó en la Reunión Anual de los Líderes del APEC realizada en Vancouver en 1997, y que se prolongó hasta el año siguiente, en la Reunión Anual de los Líderes de Kuala Lumpur, Malasia. Durante este período, el APEC intentó vanamente avanzar en forma paralela a las Metas de Bogor mediante la liberalización sectorial anticipada, o Early Voluntary Sectorial Liberalization (EVSL), por su sigla en inglés ${ }^{17}$. Esta iniciativa, que consistía en adelantar la liberalización de los sectores en que se lograran consensos, alteraba un principio básico del APEC, según el cual la liberalización unilateral y

${ }^{17}$ Jaime Lagos Erazo expone detalladamente esta fase en "Una década del Foro de Cooperación Económica del Asia Pacífico (APEC) y la participación chilena”, Santiago, DIRECON, 1999. Ver también Juan Salazar, op. cit.; pp. 210-219; y Mario Ignacio Artaza, "Chile en APEC: Aprovechando lo Mejor de la Globalización”, Diplomacia, No 88, julio-septiembre 2001; pp. 93-109. 
concertada se efectuaría sobre la base de la cobertura integral de todo el universo de bienes y servicios, para permitir márgenes de negociación a las economías más pequeñas y menos desarrolladas, a la imagen de las negociaciones multilaterales ${ }^{18}$.

El APEC se revitalizó bajo la conducción de Nueva Zelandia, en 1999. Ese año, el foro apostó primero en favor de un nuevo impulso al proyecto inicial de Acción Unilateral Concertada, mediante el perfeccionamiento de los IAP, y reiterando su compromiso con el lanzamiento de una nueva ronda multilateral de negociaciones comerciales, la llamada Ronda del Milenio, con ocasión de la Conferencia Ministerial de la OMC, que se realizaría semanas después en Seattle.

En el primer ámbito, los objetivos neocelandeses se lograron en buena medida al acordarse mejorar la transparencia y especificidad de los IAP, luego de analizar los resultados de una evaluación interna del APEC junto a otra realizada por el Consejo de Cooperación Económica del Pacífico (PECC). Se acordó, además, elaborar un conjunto de principios sobre políticas de competencia y reforma reguladora, que permitirían a cada economía adoptarlos para asegurar la fortaleza del funcionamiento de los mercados. Respecto a la fallida Ronda del Milenio, los Líderes establecieron que ésta debía incluir el tema de los aranceles de los bienes industriales junto con la agenda agrícola que dejó inconclusa la Ronda Uruguay, además de tomar debida considera- ción de los obstáculos que enfrentan las economías en desarrollo.

\section{Para asegurar el buen funcionamiento de los mercados se acordó elaborar un conjunto de principios sobre políticas de competencia y reforma reguladora.}

Además, diversas propuestas formuladas durante el año de Nueva Zelandia dieron impulso a la profundización de la agenda de trabajo del APEC. Estados Unidos promovió la incorporación de los temas de transparencia en las compras del sector público, comercio electrónico (congelamiento o no-aplicación de gravámenes al comercio realizado electrónicamente) y facilitación comercial. Economías como Chile, Japón, Filipinas, Corea del Sur y México promovieron la inclusión de los temas de inversiones, políticas de competencia y regímenes antidumping.

En Brunei, en 2000, los Líderes del APEC llamaron nuevamente a emprender una ronda de negociaciones multilaterales, con arreglo a los criterios mínimos definidos en la Reunión de Ministros de Comercio de APEC, realizada en Darwin ese mismo año. Encomendaron la adopción de una agenda equilibrada y amplia que respondiera a los intereses y planteamientos de todos los miembros. Asimismo, resolvieron emprender esfuerzos que permitieran avances sustantivos

${ }^{18}$ Juan Gabriel Valdés, "Diez años del APEC: Proyección diplomática y comercial”, Diplomacia, No 80, julio-septiembre 1999; pp. 21-25 
en las negociaciones sobre agricultura y servicios y continuar con el trabajo preparatorio en materia de tarifas industriales ${ }^{19}$.

Síntoma del estancamiento multilateral, los ministros encargados del comercio del APEC, reunidos en Shanghai, la capital financiera de China, en 2001, no pudieron lograr consensos que les permitieran acudir con un pie propositivo a la Reunión Ministerial de la OMC que se realizaría semanas después en Doha, Qatar, pese a que Singapur, Australia y Japón flexibilizaron sus posiciones y aceptaron dejar un margen para que se definiera posteriormente la agenda de la eventual nueva ronda. En medio de un clima de incertidumbre económica mundial, los líderes de APEC acordaron respaldar las negociaciones en el marco de la Ronda de Doha, y abogaron en especial por la eliminación de los subsidios a las exportaciones agrícolas. Subrayaron, asimismo, la necesidad de implementar satisfactoriamente un CAP para reducir en $5 \%$ los costos de transacción del intercambio transpacífico para el año 2006. En Shanghai, por último, se establecieron las que se denominaron "pathfinder initiatives", o iniciativas basadas en aquellos temas en que un grupo de economías miembros inician o ponen en práctica arreglos de cooperación, sin necesidad de consenso, en la medida en que sean compatibles con las metas de Bogor y se ajus- ten a los principios de voluntarismo, comprensividad, adopción de decisiones por consenso, flexibilidad, transparencia, regionalismo abierto y calendarios diferenciados según si se trate de economías desarrolladas o de economías en desarro1lo. Las economías que no estén preparadas pueden unirse a estas iniciativas cuando lo deseen ${ }^{20}$.

\section{Los ministros de comercio no pudieron lograr consensos que permitieran acudir con pie propositivo a la reunión de Doha.}

\section{La agenda de seguridad}

La Cumbre Anual de los Líderes, realizada en Shanghai a pocas semanas de los atentados contra las Torres Gemelas y el Pentágono, marcó también un punto de inflexión para la agenda del foro. Cerró un ciclo, que se inició en Auckland, en 1999, a raíz de la crisis de Timor, y a lo largo del cual se incluyeron con visibilidad cada vez mayor los temas relativos a la seguridad regional. En esa oportunidad, los líderes formularon por primera vez una declaración de índole política, al condenar los ataques terroristas y acordar medidas de cooperación para combatirlo en varias áreas ${ }^{21}$.

${ }^{19}$ Estados Unidos, Nueva Zelandia, Brunei, Singapur y Chile también anunciaron un importante acuerdo de cielos abiertos, con lo cual el movimiento aéreo, la frecuencia de vuelos, las tarifas y los tipos de aeronaves quedarán liberalizadas para las compañías aéreas de los países firmantes.

${ }^{20}$ Mario Artaza Rouxel, “APEC en diez preguntas y respuestas”, Santiago, DIRECON, 2003, p. 6.

${ }^{21}$ Idem, p. 6. 


\section{Se intercambiaron propuestas para reforzar la seguridad cibernética.}

En México, al año siguiente, con el atentado en Bali y la toma de rehenes en un teatro de Moscú como telón de fondo, los líderes suscribieron la Declaración de Los Cabos contra el terrorismo y acordaron un CAP que garantizara la seguridad del transporte y de la comercialización del flujo de bienes que se transa a lo ancho de la región. Se adoptaron tres iniciativas principales, entre las que destaca la llamada iniciativa STAR (por la sigla en inglés correspondiente a "Comercio seguro en la región del APEC"), que apunta a mejorar el movimiento seguro de contenedores mediante sistemas de seguimiento satelital y a aplicar procedimientos de certificación de seguridad a las cadenas productivas, con el objeto de garantizar que no generen riesgos productos ni procesos. Esta iniciativa también incorporó medidas relacionadas con el movimiento de personas. En segundo lugar, los ministros de finanzas del APEC discutieron un CAP para luchar contra el financiamiento del terrorismo mediante medidas que impidan el acceso de los terroristas al sistema financiero mundial. Por último, se intercambiaron propuestas para reforzar la seguridad cibernética ${ }^{22}$. Las economías en desarrollo, entre las cuales figuró Chile, resaltaron la necesidad de reforzar la cooperación para permitir que los países en desarrollo accedan a los recursos tecnológicos, financieros y humanos necesarios para aplicar las nuevas medidas de seguridad, sin perjudicar su desarrollo exportador.

\section{REGIONALISMO TRANSPACÍFICO Y TLC}

Aunque las acciones emprendidas en Nueva Zelandia contribuyeron a revitalizar el foro, estimamos que el principal factor que ayudó a salir de la fase descendente fue la concreción en Auckland de un giro que se estaba incubando en la percepción de varios ministerios de comercio y cancillerías asiáticas, además de Canberra, y que consistía en validar, en principio, la tesis sostenida por Chile junto a otras economías de la región (en particular: México y Singapur, además de los Estados Unidos), de que era posible cumplir con las Metas de Bogor mediante los TLC. Aun antes de la fallida Conferencia Ministerial de la $\mathrm{OMC}$ de Seattle, adquirieron mucha relevancia y visibilidad las negociaciones bilaterales o subregionales de TLC.

Tradicionalmente, las economías asiáticas y Australia han postulado que se deben privilegiar las negociaciones comerciales multilaterales $\mathrm{y}$, al interior del APEC, los IAP y los CAP, ya que son los principales instrumentos para lograr la liberalización unilateral y concertada. Japón, por ejemplo, ha propuesto fortale-

\footnotetext{
${ }^{22}$ Juan José Ramirez Bonilla desarrolla un análisis crítico de la irrupción de los temas de seguridad en la agenda de APEC en "APEC Hoy: Refuncionalización o Bancarrota", trabajo presentado en el marco de la II Jornada de Triangulación España, América Latina-Asia, organizada por la Casa de Asia, Barcelona, 28 de noviembre de 2002. Ver también Mario Ignacio Artaza, "Seguridad Colectiva en APEC", El Mostrador, 27 de enero, 2002 (www.elmostrador.cl).
} 
cer la Secretaría del APEC con el objeto de aumentar la transparencia del ejercicio de los IAP $^{23}$. Estados Unidos, en el otro extremo, es proclive a poner énfasis en la necesidad de generar nuevos mecanismos, junto con fijar etapas y plazos, para revigorizar los esfuerzos por cumplir con las Metas de Bogor. Así, en 2001 lideró la propuesta de la "Carta de Shanghai" de revisar la Agenda de Acción de Osaka para actualizar los mecanismos del APEC. En el noreste de Asia, Corea del Sur, tradicionalmente cerrada al uso de los TLC como instrumento de política comercial, modificó los principios de su política económica internacional y manifestó su voluntad de alcanzar acuerdos que les permitieran hacer frente a la competencia en el este de Asia y en el hemisferio americano. Al amparo de las conversaciones realizadas al margen de las reuniones formales, en Auckland surgió una serie de iniciativas para entablar negociaciones para un TLC o establecer grupos de estudio encargados de sondear las perspectivas de eventuales negociaciones. El presidente coreano, Kim Dae-jung, junto con su colega chileno, Eduardo Frei, anunciaron el inicio de negociaciones encaminadas a suscribir un TLC. A esta iniciativa se sumó la propuesta de Nueva Zelandia en favor de un Acuerdo de Sociedad Económica Amplia o Comprehensive Economic Partnership Agreement (CEPA) -entre Nueva Zelandia y Austra- lia, por una parte, y la Asociación de Naciones del Sudeste Asiático, ASEAN, por la otra. Frente a estas nuevas realidades, el APEC acordó realizar un estudio que analizara el impacto de un área de libre comercio el año 2010, y que cubriría, además de bienes y servicios, temas como compras de gobierno, inversiones, competencia, normas antidumping y de conformidad técnica, barreras no arancelarias y subsidios a las exportaciones.

\section{Se advirtió que el crecimiento explosivo y desordenado de los acuerdos de libre comercio bilaterales podía generar tendencias de fragmentación de la economía regional.}

Algunos especialistas interpretaron estos cambios como el reflejo de cierta desilusión ante la evolución del proceso APEC, advirtiendo que un crecimiento explosivo y desordenado de TLC bilaterales y subregionales podía generar tendencias de fragmentación de la economía regional, incrementando en definitiva los costos de transacción en el comercio y las finanzas transpacíficas. Éstas se verían diluidas en la medida en que se generara un proceso de fusión de los TLC, y la conclusión de buenos acuerdos comerciales amplios en el noreste Asiático ${ }^{24}$.

\footnotetext{
${ }^{23}$ Ver Juan José Ramirez Bonilla, "Enfoques interregionales sobre la globalización: Las experiencias de Asia del Este y de América Latina", Integración y Comercio, № 12, Año 4, septiembre-diciembre 2000; pp., 5-22, y Akio Hosono, "Integración económica en Asia Pacífico: Experiencias y nuevas iniciativas", Integración y Comercio, No 12, Año 4, septiembre-diciembre 2000; pp., 141-157.

${ }^{24}$ Scollay, op. cit., 2000; pp. 31-32.
} 


\section{La crisis asiática reveló la dificultad de competir con} productos de alta tecnología europeos y norteamericanos.

En realidad, la crisis asiática de 19971998 reflejó la alteración de las bases del sistema económico internacional, diluyendo las condiciones externas que permitieron el éxito del modelo asiático de desarrollo: abundancia de contingentes de trabajadores disciplinados, mercados externos en expansión, aprovechamiento de los ciclos de los productos manufacturados para avanzar en las cadenas globales de producción y abundancia de capitales para financiar la industrialización exportadora. El nuevo escenario dejó al descubierto rasgos obsoletos del modelo de política industrial, particularmente en el sistema financiero y la concentración de la estructura industrial. Reveló también en toda su magnitud el impacto de la competencia china y la dificultad de competir con los productos de alta tecnología europeos y norteamericanos. La nueva lectura que los líderes se vieron forzados a adoptar se reflejó en la política comercial, que se apartó manifiestamente de sus postulados básicos. Fue preciso reevaluar su rol como mecanismo al servicio del desarrollo industrial exportador, profundizando la apertura y la desregulación, y debió admitirse la necesidad de incorporar a los TLC como variante estratégica.
Así, los Líderes del APEC, reunidos en Brunei Darussalam en 2000, reconocieron que las iniciativas en materia de TLC eran potencialmente positivas para la liberalización del comercio y las inversiones. Ese año, los ministros de comercio, reunidos en Darwin, resaltaron la necesidad de que los TLC que se negociaran al interior de la región del APEC fuesen compatibles con la OMC y que contribuyeran a fortalecerla. Asimismo, debían aportar al cumplimiento de las Metas de Bogor y no dañar el comercio al interior de Asia-Pacífico. Un conjunto de economías, entre las cuales figuraron los Estados Unidos, Japón, Singapur y Chile, tomaron la iniciativa de coordinar un intercambio de información sobre las negociaciones en curso, y analizar las condiciones bajo las cuales son efectivamente comprensivas, integrales y compatibles con la OMC. En esa reunión de los Líderes, el Presidente Bill Clinton anunció la decisión de iniciar negociaciones separadas con Chile y Singapur para acordar sendos TLC.

\section{VISIONES TRANSPACÍFICAS \\ DEL REGIONALISMO ABIERTO}

Conforme sostienen varios especialistas, en Asia-Pacífico imperan dos aproximaciones diferentes a los TLC y, en general, a la economía política de la apertura económica regional ${ }^{25}$. La primera se enmarca en el modelo del ALCAN, y se

${ }^{25}$ Además del citado artículo de Feinberg, Stephan Haggard hace una completa revisión de los elementos políticos, económicos y sociológicos que diferencian la economía política de la integración en el hemisferio americano de aquélla del este de Asia en: "The Political Economy of Regionalism in 
basa en una intervención gubernamental limitada a la reglamentación detallada de la apertura de todos los sectores económicos incluidos, así como de los regímenes de solución de controversias y reglas de origen. En este marco, los mecanismos de mercado deben operar libremente en el contexto del nuevo espacio económico abierto por el TLC. Por otra parte, en Asia y Oceanía surge un modelo de acuerdo de última generación, los CEPA, cuya primera versión fue el acuerdo entre Australia y Nueva Zelandia (1983), posteriormente replicado en el acuerdo entre ambos países oceánicos y la ASEAN, y en el acuerdo alcanzado por Japón con Singapur, y Australia con Singapur, ambos en 2002. Estos se concentran en temas no arancelarios, aunque no los excluyen, y comprenden la creación de capacidad y la cooperación intergubernamental en los ámbitos económico y político, como mecanismo para suprimir las diferencias entre los asociados. Según Juan José Ramírez Bonilla, estos dos modelos son la concreción de estas dos concepciones radicalmente diferentes del desarrollo económico y de la participación de las economías nacionales en el proceso de globalización ${ }^{26}$. La primera responde a la filosofía liberal, en tanto que la segunda a un modelo nacional de desarrollo en que el Estado conduce estratégicamente la integración internacional en estrecha alianza con los conglomerados económicos, que incluye al mercado como instrumento idóneo, pero más limitado. Sin embargo, la versión asiática del regionalismo abierto propone proyectar la apertura lograda en el marco de las Metas de Bogor de manera automática a las economías que no pertenecen al APEC, mediante la aplicación de la cláusula de la nación más favorecida de la OMC. Esta propuesta, favorecida por economías que centran la apertura y la reforma de su política comercial en el plano multilateral, como Japón y Corea del Sur, sostiene que la OMC puede proporcionar el proceso o los mecanismos, mientras que el APEC ofrece las metas.

\section{De acuerdo con el modelo del NAFTA, los mecanismos de mercado deberían operar libremente en el marco del espacio económico abierto por el tratado.}

Como hemos señalado, entre las economías asiáticas desarrolladas, que sólo recientemente han pasado a formar parte del grupo de países que han adherido a los TLC, validándolos en ciertas circunstancias como mecanismos idóneos para la liberalización del comercio y de las finanzas internacionales, destacan Corea del Sur y Japón. China, cuyo ingreso a la OMC recién tuvo lugar en 2001, es un caso de economía de alto crecimiento pero en transición, y China Hong-Kong y China-Taipei, debido al factor político, también, aunque como entidad económica,

Asia and the Americas", Mansfield, Edward D. y Helen V. Milner, The Political Economy of Regionalism, Nueva York, Columbia University Press, 1997.

${ }^{26}$ Ramírez, op. cit., 2002; p. 6. 
China-Hong Kong haya concluido un TLC con China el 2003. Conviene entonces analizar más en detalle a Japón y Corea del Sur, para entender los cambios que están operando en la economía política de la apertura comercial transpacífica. Posteriormente analizaremos también el impacto que tiene la iniciativa conocida como ASEAN + 3 .

\section{Corea se apartó de la línea de apoyo exclusivo a las negociaciones multilaterales.}

\section{a) Corea del Sur}

Corea del Sur fue la primera economía del noreste asiático que adoptó nuevos lineamientos comerciales en los cuales tuvieron cabida los TLC. La nueva política se gestó bajo la administración de Kim Young-sam (1993-1998), pero se adoptó bajo la presidencia de Kim Daejung (1998-2003). Ésta se apartó de la línea tradicional de apoyo exclusivo a las negociaciones multilaterales y optó por una estrategia de dos carriles: en favor de la profundización de la liberalización del intercambio multilateral mediante una nueva ronda de negociaciones al amparo de la OMC, pero abriendo el juego a través de una ofensiva en el plano bilateral, con el objetivo a largo plazo de suscribir TLC con los bloques más importantes.

Los principales factores que provo- caron este giro de la política comercial coreana fueron, primero, la reemergencia del regionalismo en la década de los noventa y el consiguiente miedo a quedar fuera de las regiones formadas en torno a las economías más dinámicas del sistema mundial. En segundo lugar, la crisis asiática forzó a las autoridades a profundizar la apertura de la economía coreana bajo los dictados del FMI, y convenció a más sectores estratégicos de la burocracia, pero también de la clase política, acerca de la necesidad de abrirse al comercio y a los mercados financieros internacionales y sobre los beneficios derivados de ello ${ }^{27}$.

Como señaláramos, con ocasión de la Cumbre de los Líderes de APEC, realizada en 1999 en Auckland, los presidentes de Corea y de Chile anunciaron el inicio de las negociaciones para suscribir un acuerdo de libre comercio, las que finalmente serían dificultosas y concluirían en 2002. Corea optó por negociar con Chile en el marco de una estrategia consistente en establecer, primero, acuerdos con "pequeños países estratégicos" como etapa previa a la celebración de acuerdos con sus principales socios comerciales (Japón, Estados Unidos y China). Chile representaba apenas el $0,5 \%$ de las exportaciones coreanas y el $1,0 \%$ de sus importaciones, reduciendo los costos internos de ajuste. Favoreció a Chile, además, su condición de economía complementaria especializada en las exportaciones de recursos naturales (cobre y productos forestales primarios) y en la importación de bienes manu-

${ }^{27}$ Hernán Gutiérrez B., "Corea en los '90: Las estrategias de las economías asiáticas industrializadas ante la globalización”, Estudios Internacionales, Año XXXIV, № 134, abril-junio 2001; pp. 57-73. 
facturados, principalmente vehículos y productos electrónicos. Por último, la condición del país sudamericano como uno de los más activos en materia de liberalización unilateral y negociación de TLC aseguraba un acuerdo comercial relativamente amplio y sin el lastre de numerosos acuerdos laterales ${ }^{28}$. A pesar de ello, la reticencia coreana a liberalizar el acceso en los sectores agrícola, forestal y de pesca dificultó las negociaciones. El TLC acordado finalmente con Chile tiene limitada incidencia en el comercio agrícola y al cabo de 4 años, $47 \%$ de todas las exportaciones chilenas ingresarán a Corea del Sur con arancel cero, en comparación con el $85 \%$ logrado por este país en el TLC con los Estados Unidos, o el $89 \%$ logrado en el TLC con la Unión Europea. Aun así, la fuerte oposición de los productores agrícolas coreanos ha impedido, hasta la fecha (diciembre de 2003) la ratificación por el Parlamento del TLC con Chile.

\section{El tratado de libre comercio acordado con Chile tiene efectos limitados en el comercio agrícola.}

\section{b) Japón}

Tokio siempre había privilegiado las negociaciones multilaterales y visto con desconfianza los TLC, por cuanto estimaba que conducirían a formar espacios económicos regionales rivales. Sin embargo, desde las oficinas de influyentes agencias niponas y de los cuarteles generales de los grandes conglomerados, empezó a verse a Japón como eje de un TLC asiático en el futuro cercano, centrado en el comercio pero sobre todo en las inversiones, con conexiones con el NAFTA y el Cono Sur ${ }^{29}$.

Es así como en 1999 Japón recogió la propuesta coreana de formar grupos binacionales de expertos para estudiar el impacto de un eventual TLC y pocos meses después hizo lo propio con las autoridades mexicanas. En el marco de la XIX ${ }^{\mathrm{a}}$ Reunión Anual del Comité Empresarial Chile-Japón, realizada en Tokio en el mes de mayo de 2000, representantes del sector privado japonés propusieron a las autoridades de ambos países realizar un estudio similar, el que finalmente llevaron a cabo en forma conjunta expertos de la Oficina de Comercio Exterior de Japón -JETRO, según su sigla en inglés- $\mathrm{y}$ de la Dirección General de Relaciones Económicas Internacionales (DIRECON) de la Cancillería chilena. El nuevo interés de Japón quedó también de manifiesto en la Reunión de Altos Funcionarios de APEC, realizada en Brunei Darussalam, cuando propuso que se hiciera un CAP para evaluar la contribución de los TLC a la apertura de la economía regional e internacional.

Los nuevos lineamientos de la políti-

${ }^{28}$ Inkyo Cheong, Korea-Chile FTA: Korea's Position, Seúl, Korean Institute for International Economic Policy, 1999.

${ }^{29}$ Ver Hernán Gutiérrez B., "Chile y Japón: Hacia una Nueva Asociación Estratégica”, Diplomacia No 88, julio-septiembre 2001; pp. 117-130. 
ca japonesa en materia de TLC se concretaron en el Libro Blanco sobre el Comercio Internacional, de 1999, elaborado por el Ministrio de Comercio Internacional y de Industria (MITI), y en el cual se reconocen los aspectos positivos de los TLC y se aboga por una respuesta japonesa constructiva y flexible. A la fecha, Japón sólo ha suscrito, en 2001, un TLC con Singapur, que se enmarca en el modelo de los CEPA y que excluye ciertos productos agrícolas. Las negociaciones con México han sido dificultosas y se han demorado más de lo que se esperaba. La JETRO ha realizado un estudio sobre los efectos de un TLC conjuntamente con el Instituto Coreano de Política Económica Internacional (KIEP), iniciándose en 2002 la fase de consultas privadas, al alero de la Federación de Organizaciones Económicas de Japón (Keidanren), principal asociación del sector privado, claramente favorable a los acuerdos ${ }^{30}$. A la fecha, sin embargo esta iniciativa está estancada. Con Chile, por último, en el primer semestre de 2001 se concluyeron los estudios conjuntos realizados por la DIRECON y JETRO, pero no se ha avanzado hacia etapas más formales debido a la prioridad asignada por Tokio al TLC con México y a las negociaciones con la ASEAN y Corea del Sur. Otros países, incluyendo Australia y Canadá, por último, han expresado también su interés en generar un nuevo marco para sus relaciones económicas con Japón, pero no se han registrado avances significativos.

\section{LOS TLC Y EL NUEVO ESCENARIO IN- TERNACIONAL E INTERNO}

Los principales elementos que explican esta nueva lectura asiática de la apertura concertada vía TLC se encuentran en el nuevo escenario de la economía internacional que enfrentan. En primer lugar, se han multiplicado las negociaciones de TLC, con una clara tendencia a ordenarse en torno a regiones, salvo en el noreste de Asia. En su conjunto, estos TLC producen una significativa desviación del comercio en detrimento de las exportaciones de productos japoneses y coreanos. Además, dado que los TLC abarcan más que las preferencias arancelarias e incluyen temas que configuran un espacio de negocios más integrado y armónico, Japón y Corea del Sur pierden posiciones relativas en los sectores más dinámicos de la economía global, como servicios, telecomunicaciones y transporte.

\section{Salvo en el noreste de Asia, las negociaciones de acuerdos de libre comercio tienden a ordenarse en torno a regiones.}

Las iniciativas adoptadas por Japón y Corea del Sur revelan también objetivos específicos en cada caso. Por una parte, un eventual TLC de Japón con México responde a la necesidad de mantener el acceso al mercado norteamericano en el contexto del desmantelamiento del régi-

${ }^{30}$ Japan Federation of Economic Organizations (Keidanren), Urgent Call for Active Promotion of Free Trade Agreements. Towards a New Dimension in Trade Policy, Tokio, Keidanren, julio de 2000. 
men de maquila, además de contrarrestar la desviación de comercio que genera el NAFTA junto al TLC de México con la Unión Europea. Por otra parte, el TLC entre Japón y Singapur y un eventual TLC de Japón con Chile responden a consideraciones estratégicas: acuerdos con economías pequeñas, muy abiertas a la economía internacional y que surgen como plataformas de negocios regionales debido a su transparencia y estabilidad. Sirven también como ejercicios conducentes al aprendizaje en materia de negociaciones y a la generación de consensos y apoyos internos para la liberalización de los sectores sensibles.

\section{La traumática experiencia de la crisis financiera estimuló una lectura y práctica renovadas del regionalismo.}

Las causas del cambio de la política comercial son tanto de orden interno como internacional. En ciertos círculos privados y estatales, el estancamiento de la economía japonesa en los últimos años ha generado consensos en el sentido de que se debe proceder a una profunda reforma del sistema económico, en la mayoría de los casos para lograr una mayor apertura a la economía internacional, tanto en el sector productivo como en el financiero $^{31}$. Los TLC, a falta de avances concretos en el marco de la OMC, permiten consolidar las reformas internas ${ }^{32}$.

\section{ASEAN + 3}

De fructificar las iniciativas de integración que se están articulando en torno al eje compuesto por la ASEAN y que involucran a China, Japón y Corea del Sur, la economía política de Asia Pacífico estaría en la antesala de un cambio fundamental. En noviembre de 2001, los jefes de gobierno de China y de la ASEAN acordaron crear un nuevo marco de cooperación económica a partir de un área de libre comercio, que se concretaría en un plazo de diez años. Pocos meses después, durante una gira oficial por la región, el Primer Ministro japonés, Junichiro Koizumi propuso a los miembros de ASEAN un CEPA ${ }^{33}$. De concretarse estas iniciativas, se despejaría un nuevo escenario en el este de Asia. La región se proyectaría como un bloque en la economía internacional, consolidando el proceso de integración que se inició en los años ochenta, estimulado por la inversión intrarregional y el crecimiento del comercio intraindustrial. China, que ya ha suscrito un CEPA con China-Hong Kong, ha avanzado más que Japón y Corea del Sur, demostrando una voluntad política que hace creíble su iniciativa, a pesar de las

31 "La Nueva Política de Japón ante el Acuerdo de Libre Comercio", Cuadernos de Japón, Vol. IV, $\mathrm{N}^{\mathrm{o}} 1,2001$.

${ }^{32}$ Tanaka Hitoshi señala que Japón "debe ser capaz de conseguir dos cosas al mismo tiempo: una economía más eficiente mediante reformas estructurales y encarar el problema estructural del envejecimiento de la población”, en: “¿Puede Japón salir adelante?”, Cuadernos de Japón, Vol. XIV, Nº 1, 2001, p. 9.

${ }^{33}$ Boletín Fundación Chilena del Pacífico, $\mathrm{N}^{\mathrm{o}} 2$, julio 2002, <http://www.funpacifico.cl $>$. 
reticencias de algunas economías de la $\mathrm{ASEAN}^{34}$, y de los temores de una creciente competencia china, en Tokio y Seúl.

Por cierto, como ya lo hemos señalado, la traumática experiencia de la crisis financiera de 1997-1998 estimuló una renovada lectura y práctica del regionalismo en el este de Asia, refrendadas en la reunión de ASEAN + 3, en 1998. Pero igualmente importantes han sido los cambios sistémicos que se han registrado, en particular la creciente interdependencia alimentada por el crecimiento del comercio y de las inversiones intrarregionales, factores clave del crecimiento de las economías asiáticas en las dos últimas décadas. Ellos, en su conjunto, han forjado una expansiva red de relaciones comerciales, la que a su vez ha estimulado una mayor cooperación intergubernamental para facilitar las transacciones comerciales ${ }^{35}$. En 2002, el comercio entre Japón y la ASEAN superó los 114 mil millones de dólares, reduciéndose así el déficit que registraba el bloque del sudeste asiático, que exporta principalmente maquinaria y equipos electrónicos, productos minerales, agrícolas y alimentos elaborados, así como madera y manufacturas de madera. Asimismo, las relaciones económicas entre China y la ASEAN se han incrementado notablemente. En 2002 el comercio superó la barrera de los 60 billones de dólares, creciendo más de $250 \%$ desde 1996, siendo las principales exportaciones de la ASEAN maquinaria y equipos electrónicos, productos minerales, agrícolas y alimentos preparados, plástico y textiles y confección ${ }^{36}$. Más importante aún, el mercado chino les proporcionó una vía para superar la recesión, importando productos por un monto de 33.800 millones de dólares en el año 2000, más del triple del valor registrado en 1996. En el plano financiero, Japón es uno de los mayores inversionistas de la subregión, si bien el flujo de capitales ha disminuido en los últimos años a raíz de la debilidad del yen. Por otra parte, las empresas chinas, estimuladas por su gobierno, también están adquiriendo mayor relevancia como inversionistas. Aun cuando en términos absolutos la inversión china es todavía modesta, está creciendo aceleradamente: en el año 2000 Beijing aprobó nuevas inversiones en la región por un monto de 108 millones de dólares, una cifra superior en $50 \%$ a la del año anterior.

\section{En el plano financiero, Japón es uno de los mayores inversionistas de la subregión.}

${ }^{34}$ Ver, por ejemplo, John Lawrence Avila, "Looking before leaping into bilateral FTAs: Issues in Asian bilateralism", Phuket, 2003, trabajo presentado en el marco del Encuentro Anual del Consorcio Internacional de Centros de Estudios APEC, y Hadi Soesastro, "ASEAN perspectives on preferential trading arrangements", PECC Issues, agosto de 2003.

${ }^{35}$ DIRECON, Estudios Especiales, "Este de Asia: Configurando nuevos escenarios en la economía mundial", Santiago, primer trimestre, 2003, <http://www.direcon.cl $>$.

${ }^{36}$ John Lawrence Avila, "Torn between two lovers: ASEAN and its evolving economic relations with China and Japan", Mérida, 2002, trabajo presentado en el marco del Encuentro Anual del Consorcio Internacional de Centros de Estudios APEC. 
Una eventual área de libre comercio que integrara a Japón y China con la ASEAN estimularía fuertemente el comercio intrarregional. Las evaluaciones iniciales indican que las exportaciones de la ASEAN a China crecerían $48 \%$, en tanto los embarques desde China aumentarían $52 \%$. Pero, sobre todo, tendría un efecto considerable en la geografía económica de Asia-Pacífico. Un área de libre comercio entre ASEAN y China concentraría un PGB combinado de al menos 1,6 billones de dólares y, en el caso de Japón, de 5,5 billones de dólares, convirtiendo al este de Asia en un polo frente a Norteamérica y Europa.

\section{APEC ChILE-2004}

Al igual que en la economía mundial, en Asia-Pacífico los TLC son una realidad que llegó para quedarse. En los últimos años se han suscrito en la región numerosos TLC o CEPA. El año 2002 registra el CEPA celebrado entre el Área de Libre Comercio de ASEAN y Australia y Nueva Zelandia, junto a los TLC acordado por Chile con Corea del Sur, y con los Estados Unidos y Singapur, respectivamente. Desde el 2002, Canadá está negociando un acuerdo con Singapur, Australia uno con Tailandia y otro con los Estados Unidos, y México con Japón. Japón y la ASEAN anunciaron ese año futuras negociaciones, así como China con la Asociación, entidad que desde 2002 también está negociando con los Estados Unidos un Acuerdo Marco para el Comercio y las Inversiones -TIFA, por su sigla en inglés-. Nueva Zelandia inició negociaciones con China-Hong Kong ese año y anunció, junto a Chile y Singapur, negociaciones para un acuerdo trilateral, conocido como P-3 (Pácifico-3). El 2003, concluyeron las negociaciones entre China y China-Hong Kong, en tanto que los Estados Unidos y Tailandia anunciaron el inicio de negociaciones.

\section{Nueva Zelandia anunció negociaciones para un acuerdo trilateral con Chile y Singapur.}

No es de extrañar, entonces, que en la última reunión de los Líderes del APEC, efectuada en 2003 en Bangkok, se pusiera con propiedad el tema de los TLC en la agenda del foro, el que será uno de los ejes temáticos del año APEC en Chile. Meses antes, los Altos Funcionarios realizaron en Khon Kaen el primer Diálogo de Política sobre TLC. En la Declaración de Bangkok, los Líderes acordaron promover el libre comercio de una manera coordinada entre los esquemas multilaterales, regionales y bilaterales, de tal forma que sean complementarios y mutuamente potenciadores ${ }^{37}$. En las conversaciones informales, reconocieron que al interior de la región de Asia Pacífico los TLC podrían contribuir a alcanzar las Metas de Bogor, en la medida en que fue-

37 - Bangkok Declaration on Partnership for the Future", 21 de octubre, 2003, <http:// www.apecsec.org.sg>. 
ran compatibles con la OMC. Las economías que estaban en condiciones de liberalizar su comercio lo podían realizar y ayudar a las demás a avanzar, trilateralizando los TLC bilaterales, coordinando aspectos críticos de diversos TLC y trabajando por la meta de alcanzar el libre comercio regional. Los ministros ${ }^{38}$, finalmente, reconocieron que si los TLC son de caracter amplio pueden promover la liberalización competitiva en la región y revitalizar la Ronda Doha de Desarrollo, y por ello convocaron a un segundo Diálogo de Políticas sobre los TLC, a realizarse en 2004, en Pucón, Chile. Llamaron también a aprovechar el trabajo que ha adelantado en esta materia el Foro Comercial del $\mathrm{PECC}^{39}$, para desarrollar marcos que maximicen la contribución de los TLC a la consecución de las Metas de Bogor. El Comité Económico del APEC, por su parte, acordó realizar un estudio bajo la coordinación chilena, para profundizar el conocimiento de los TLC en la región del APEC.

38 "Fifteenth APEC Ministerial Meeting. Joint Statement. Summary of Key Issues", <http:// www.apecsec.org.sg>.

${ }^{39}$ Los resultados de este trabajo están sintetizados en Robert Scollay y Fernando González-Vigil, "Proposals for an APEC Common Understanding on RTAs" y "Asia Pacific RTAs an Avenue for Achieving APEC's Bogor Goals”, Singapur: PECC - Trade Forum, 2003, <http://www.pecc.org>. 